\title{
Effect of Melia azedarach (Sapindales: Meliaceae) fruit extracts on Citrus Leafminer Phyllocnistis citrella (Lepidoptera: Gracillariidae)
}

\author{
Maher M Mckenna', Efat M Abou-Fakhr Hammad² ${ }^{*}$ and Mohamad T Farran ${ }^{3}$
}

\begin{abstract}
Melia azedarach L. extracts were studied in comparison with selected biorational insecticides against the citrus leafminer Phyllocnistis citrella Stainton under field conditions. Citrus limon (L.) Burm. F. trees were exposed to: Melia extracts of green and mature fruits, Neem oil (30\% a.i.), abamectin (1.8\% a.i.) and control. Two sprays of each treatment (except for Melia mature fruit extract) were executed at 10-d intervals. The live number of the $1^{\text {st }}$ and later $\left(2^{\text {nd }} \& 3^{\text {rd }}\right.$ ) larval instars per leaf were recorded at initial sampling date and at 10-d intervals after each spray application. Results indicated that there were significant differences in the number of live larval instars among treatments. Melia extracts and the two biorationals, neem oil and abamectin, decreased the larvae population significantly to lower numbers than that of the control at 10 days after each spray application. However, the decrease caused by neem oil and abamectin was significantly higher than that of Melia extracts. Thus, these extracts might be considered as potential alternative with other biorational control methods in management of the leafminer. Further research including bioassays is needed to determine the factors responsible for reducing larvae population and whether these Melia extracts can be utilized in future citrus IPM programs as a tool for citrus leafminer management.
\end{abstract}

Keywords: Lemon, Leafminer, Botanical, Chinaberry, Biorational, Larvae

\section{Introduction}

The citrus leafminer (CLM) Phyllocnistis citrella Stainton (Lepidoptera: Gracillariidae: Phyllocnistinae) is one of the major constraints to citrus production in the world. Effective chemical control of the CLM is limited as the feeding larvae are protected by the leaf cuticle mainly in mines of immature foliage and the pupal stage is also protected by the rolled leaf margins (Jones 2001; Beattie 2004). Several pressures as pesticide resistance has accelerated the search for safe environmentally, toxicologically, selective and efficacious pesticides (Weinzierl and Henn 1991; Dias et al. 2005; Amiri-Besheli 2008, 2009). Biopesticides, including botanicals, can offer a safe and effective alternative to conventional insecticides controlling major insect pests. Botanicals are a promising source of pest control compounds. The pool of plants possessing insecticidal substances is

\footnotetext{
*Correspondence: efat@aub.edu.lb

${ }^{2}$ Department of Agricultural Sciences, Faculty of Agricultural and Food

Sciences, American University of Beirut, Beirut, Lebanon

Full list of author information is available at the end of the article
}

enormous (FAO 1992; Jacobson 1989). These have generated remarkable interest in recent years as potential sources of natural insect control agents. Over 2000 species of plants are known to possess different degrees of insecticidal activity (Jacobson 1975). However, a few plant-based pesticides have already been utilized as plant protection products (Isman 2006). Several secondary plant products represent a large reservoir of chemical structures with biological activity against pests in form of pesticides (Grainage and Ahmed 1988; Schmutterer 1990; Lowery and Isman 1994; Miller and Uetz 1998). Throughout history, plant products have been successfully exploited as insecticides, insect repellents, insect antifeedants (Lydon and Duke 1989) or as altering insect feeding behavior, growth, development, and behavior during mating and oviposition (Yang and Tang 1988).

The Chinaberry tree, Melia azedarach L. (Sapindales: Meliaceae), is a deciduous tree often grown for its medicinal uses and for shade or ornament on roadsides. The non-conventional insecticidal effects of extracts or 
compounds isolated from Meliaceae trees include: partial reduction or complete inhibition of fecundity and egg hatchability, reduction of life span of adults, oviposition deterrence, antifeedant effects, and insect growth regulatory effects at molting (Ascher 1993). M. azedarach has been shown to possess many insecticidal effects against different pest species (Shin-Foon 1987; Ascher et al. 1995; Nardo et al. 1997; Valladares et al. 1997; Jazzar and Abou-Fakhr Hammad 2003; Abou-Fakhr Hammad and McAuslane 2006, 2010; Al-Akhras 2010). A limited number of studies have dealt with the use of botanicals against the citrus leafminer. Amiri-Besheli (2011) indicated that the use of biopesticides such as Tondexir ${ }^{\circledR}$ extracts from hot pepper, discouraged CLM adults from laying eggs on leaves and posed lower risk to humans and the environment than other pesticides. Pepper extracts worked better on soft bodied insects during the larval stage as the chemical was able to penetrate the leaves. Howard (1993) also found that the 2 biorationals, Avid (abamectin $0.15 \mathrm{EC}$ ) and Azatin (60 ppm azadirachtin) with Triton B-1956 spreader-sticker (Rhom \& Haas Co., Philadelphia, PA), prevented damage by CLM when sprayed on leaves prior to oviposition by this insect; these sprays were timed just as trees were flushing.

The main strategy in plant protection scopes is to minimize the use of synthetic compounds in benefit of alternatives, such as biorational insecticides. Pesticide resistance and negative effects on non-target organisms are the main problems in pest management (FAO 1992; Franzen 1993). Natural insecticides are relatively harmless against the non-target organisms. In agricultural pest management, botanical insecticides are best suited for use in organic food production in industrialized countries but can play a much greater role in the production and postharvest protection of food in developing countries (Isman 2006). Availability of Melia trees allows growers to use available resources to control pests of significant economic importance. The objective of this study is to elucidate the efficacy of $M$. azedarach fruit extracts in management of the citrus leafminer larvae population under field conditions in comparison with other biopesticides.

\section{Materials and methods}

The experiment was conducted during the summer of 2011 in an orchard of Citrus $\times$ limon (L.) Burm.f. Monachello lemon variety (Moussa and El Hajj 2010) located in Tyre, South Lebanon. This variety provides fruits all year round, mainly in winter and spring; the fruit is medium-small, elliptical but tapering at both ends; neck lacking; nipple small and inconspicuous with few or no seeds. Fruit color is yellow at maturity with a thin rind. The tree has nearly thornless branches with dense foliage. During the experimental period (June-July), the maximum ambient temperature ranged between 28 and $32^{\circ} \mathrm{C}$ whereas the minimum temperature was $20-22^{\circ} \mathrm{C}$ (http://freemeteo. com/default.asp?pid $=15 \& l a=19 \& c n=L B)$. Sampled leaves were examined at the Plant Protection Laboratory, Faculty of Agriculture \& Veterinary Sciences, Lebanese University.

\section{Site description}

The lemon orchard is located in Tyrdebba village, 7 kilometers from city of Tyre in South Lebanon, at an altitude of 180 meters. The orchard is adjacent to an olive orchard on the North and to an orange orchard on the South side, while roads bordered East and West sides. The orchard contained 92 lemon trees, distributed over two terraces; one terrace is 2 meters higher than the other and is separated by a steep slope. Trees were 8 years old, distributed in lines, with $3 \mathrm{~m}$ spacing between and within those lines. Three years prior to the experiment, trees have been treated once with Supracide ${ }^{\circledR}$ (a.i. methidathion 25\%) while no other application of pesticides or chemical fertilizers has been given. In order to obtain the essential for the experiment availability of young flush, trees were sufficiently irrigated in late-April 2011.

\section{Preparation of M. azedarach extracts}

Four $\mathrm{Kg}$ of $M$. azedarach green fruits and $2 \mathrm{Kg}$ of Melia mature fruits were collected from trees at Tyrdebba village, washed and cleaned with tap water then rinsed with distilled water. Fruits were left to dry at room temperature for 2 days, and then crushed with a hammer into small pieces and ground using a grinding machine (BRAUN Multiquick 3, Hungary). The obtained material was soaked in distilled water, at a rate of $1 \mathrm{~g}$ per $5 \mathrm{ml}$, for 24 hours prior to spraying. The soaked material was filtered using a fine mesh $(500 \mu \mathrm{m})$ cloth material. About twenty three liters of each of Melia green and mature fruits extracts were obtained after filtration.

\section{Experimental procedure}

Four different treatments were tested: Melia green fruit extract, Melia mature fruit extract, neem oil-30\% (Atlantica Agricola, Spain), abamectin 1.8\% w/v Mectin $^{\circledR}$ 1.8 EC (Adonis Industrial - S.I.AD s.a.l, Lebanon). Five single-tree replicates per treatment were used, plus 5 trees treated with tap water used as control. Trees were surrounded by untreated border trees, selected so as to avoid spray drifting. All products were applied as foliar sprays to runoff (approximately 4-L), coating upper and lower leaf surfaces, using an 8-L pressure gun sprayer (Zhejiang CNG Plastic Co., China). Also, it's worth mentioning that in windy weather, the canopy of the control trees was covered with plastic sheet to avoid spray drifting from treated trees. Neem oil and abamectin, as two positive controls, were used at the recommended dose of 
$0.1 \mu \mathrm{l} / 100 \mathrm{ml}$ and $0.03 \mathrm{ml} / 100 \mathrm{ml}$, respectively. First application was made on June $16^{\text {th }}$ and the second on June $26^{\text {th }}$. In the second application, 3 treatments plus the control were tested, because there was no sufficient Melia mature fruit extract.

\section{Data collection}

Three samplings were made. The first sampling was just before the first spray application, the second sampling was10 days after the first application and before the second spray, and the third sampling was10 days after the second application. In each sampling, 24 young leaves were collected from each tree; 6 from each geographical direction (North, South, East, and West), 3 from the inner and 3 from the outer part of the canopy. The latter factors were selected in order to take representative sample from each tree-replicate. Totally, 600 leaves were collected in each of the first two samplings and 480 in the third. Leaves collected from each direction and tree location were placed in separate labeled small plastic bags and kept transferred within a cooler to the Laboratory, in which they were stored at $10^{\circ} \mathrm{C}$ until their observation. Numbers of live 1st instar (L1) and 2nd \& 3rd instar larvae (L2 \& L3) per leaf were recorded. The length of the larva was used for separation of the CLM larval instars; $1^{\text {st }}$ instar larvae were less than $2 \mathrm{~mm}$ in length whereas the $2^{\text {nd }}$ and $3^{\text {rd }}$ instars were between 2 and $3 \mathrm{~mm}$; in addition to their length, the latter instars were translucent and yellowish-green (Kerns et al. 2001).

\section{Statistical analysis}

The experiment was laid out as a three-way factorial arrangement of treatments $(5 \times 4 \times 2)$ for treatment, geographical direction and leaf location, respectively along with their two- and three-way interactions in a complete randomized design. As there was no significant interaction among the 3 factors, the data were pooled and analyzed as a one-way ANOVA with the treatment as the main factor. Consequently, there were 5 treatments (only 4 treatments in the third sampling) with 5 treereplicates per treatment. The average numbers of live larvae per leaf were used in the data analysis, after ensuring their normal distribution by transforming the data using sqrt $(x+1)$ with $x$ being the number of live larvae per leaf (Gomez and Gomez 1984). The analysis of data was performed using the general linear models procedure, and means were separated by Student Newman Keuls test whenever $\mathrm{P}<0.05$ was detected (SAS 1992).

\section{Results}

None of the interaction terms among the three main factors: treatment, geographical direction and leaf location was significant. In addition, the factorial analysis showed that direction and location were not significant indicating that CLM had no preference for inner or outside flush of citrus trees nor has preference for infesting one of the directions of the tree. Consequently, data were pooled as indicated above and analyzed with the treatment being the main factor, with mean values presented in Tables $1 \& 2$. There were no significant differences in the initial number of live larvae of all instars at the first sampling date before applying the $1^{\text {st }}$ spray of each treatment. The initial counts were comparable among treatments with a range of 0.333-0.367 and 0.383-0.492 larva per leaf for the L1 and (L2 \& L3) larval instars, respectively.

There were significant differences in number of L1 among treatments at 10 days after the $1^{\text {st }}$ spray application $(\mathrm{F}=76.77 ; \mathrm{df}=4,24 ; \mathrm{p}<0.0001)$ and at 10 days after the $2^{\text {nd }}$ spray application $(\mathrm{F}=104.66 ; \mathrm{df}=3,19 ; \mathrm{p}<0.0001)$. Similarly, there were significant differences $(F=89.02$; $\mathrm{df}=4,24 ; \mathrm{p}<0.0001)$ and $(\mathrm{F}=58.53 ; \mathrm{df}=3,19 ; \mathrm{p}<0.0001)$ in number of L2 \& L3 combined among treatments at the previously mentioned dates of post spray applications, respectively.

There was no significant difference in number of live larvae per leaf between Melia green and mature fruit extracts, but both treatments were significantly different from the control. As shown in Table 1, the Melia green and mature fruit extracts have caused a significantly low population of L1 (0.342 and 0.317 live larvae per leaf, respectively) compared with the control (0.517) at 10 days after $1^{\text {st }}$ spray application. Also in comparison with the control, the Melia green fruit extract resulted in a significantly lower L1 population (0.400 Vs 0.658 live larvae per leaf) at 10 days after the $2^{\text {nd }}$ spray application. Similarly, the Melia green and mature fruit extracts have maintained a significantly low population of the L2 \& L3 combined $(0.358$ and 0.350 live larvae per leaf, respectively) in comparison with the control (0.567) at 10 days

Table 1 Effects of Melia azedarach fruit extracts and selected biorational insecticides on Citrus leafminer first instar larvae (L1) infesting canopy of Lemon trees under field conditions

\begin{tabular}{llll}
\hline \multirow{2}{*}{ Treatments } & \multicolumn{2}{l}{ Average number of live larvae (L1) per leaf* } \\
\cline { 2 - 4 } & $\begin{array}{l}\text { Before } \\
\text { 1st spray** }\end{array}$ & $\begin{array}{l}\text { 10 days after } \\
\text { 1 st spray }\end{array}$ & $\begin{array}{l}\text { 10 days after } \\
\text { 2nd spray }\end{array}$ \\
\hline Control & $0.330 \pm 0.018$ & $0.517 \pm 0.023 \mathrm{a}$ & $0.658 \pm 0.020 \mathrm{a}$ \\
$\begin{array}{l}\text { Melia green } \\
\text { fruit extract }\end{array}$ & $0.330 \pm 0.018$ & $0.342 \pm 0.018 \mathrm{~b}$ & $0.400 \pm 0.019 \mathrm{~b}$ \\
$\begin{array}{l}\text { Melia mature } \\
\text { fruit extract }\end{array}$ & $0.350 \pm 0.016$ & $0.317 \pm 0.019 \mathrm{~b}$ & $-\mathbf{- -}$ \\
Neem oil & $0.342 \pm 0.017$ & $0.100 \pm 0.011 \mathrm{C}$ & $0.083 \pm 0.010 \mathrm{c}$ \\
Abamectin & $0.367 \pm 0.016$ & $0.058 \pm 0.009 \mathrm{C}$ & $0.067 \pm 0.010 \mathrm{C}$ \\
\hline
\end{tabular}

Measurement values presented are mean + SEM.

* = Means in a column followed by different letters are significantly different (Student Newman Keuls test, $\mathrm{P}<0.05$ ).

** = Initial counts of live larvae were not significantly different at the first date of the experiment before applying the $1^{\text {st }}$ spray for each treatment. 
Table 2 Effects of Melia azedarach fruit extracts and selected biorational insecticides on Citrus leafminer $2^{\text {nd }} \& 3^{\text {rd }}$ instar larvae (L2\& L3) infesting canopy of Lemon trees under field conditions

\begin{tabular}{llll}
\hline \multirow{2}{*}{ Treatments } & \multicolumn{3}{l}{ Average number of live larvae (L2\&L3) per leaf* } \\
\cline { 2 - 4 } & $\begin{array}{l}\text { Before } \\
\text { 1st spray** }\end{array}$ & $\begin{array}{l}\text { 10 days after } \\
\text { 1st spray }\end{array}$ & $\begin{array}{l}\text { 10 days after } \\
\text { 2nd spray }\end{array}$ \\
\hline Control & $0.467 \pm 0.018$ & $0.567 \pm 0.022$ a & $0.850 \pm 0.026 \mathrm{a}$ \\
$\begin{array}{l}\text { Melia green } \\
\text { fruit extract }\end{array}$ & $0.492 \pm 0.018$ & $0.358 \pm 0.017 \mathrm{~b}$ & $0.417 \pm 0.019 \mathrm{~b}$ \\
$\begin{array}{l}\text { Melia mature } \\
\text { fruit extract }\end{array}$ & $0.492 \pm 0.017$ & $0.350 \pm 0.017 \mathrm{~b}$ & --- \\
Neem oil & $0.475 \pm 0.018$ & $0.108 \pm 0.012 \mathrm{C}$ & $0.100 \pm 0.011 \mathrm{c}$ \\
Abamectin & $0.383 \pm 0.020$ & $0.050 \pm 0.010 \mathrm{C}$ & $0.033 \pm 0.007 \mathrm{C}$ \\
\hline
\end{tabular}

Measurement values presented are mean + SEM.

* = Means in a column followed by different letters are significantly different (Student Newman Keuls test, $\mathrm{P}<0.05$ ).

${ }^{* *}=$ Initial counts of live larvae were not significantly different at the first date of the experiment before applying the $1^{\text {st }}$ spray for each treatment.

after the $1^{\text {st }}$ spray application (Table 2 ). In addition, the Melia green fruit extract had also maintained a low population of the L2 \& L3 (0.417) as compared with 0.850 larvae per leaf for the control at 10 days after the $2^{\text {nd }}$ spray application.

In this study, the Melia extracts seem to keep the population of the CLM at a relatively constant level rather than increasing its larval numbers as in the control; the latter showed increased population of the CLM larval number throughout the experiment (Tables $1 \& 2$ ). The two commercial insecticides: Neem oil and abamectin had comparative CLM results irrespective of the spray application dates. They had, however, a significantly higher effect than the two Melia fruit extracts. In fact, the two insecticides significantly reduced the population of L1 to 0.100 and 0.058 and L2 \& L3 (combined) to 0.108 and 0.050 larvae per leaf, respectively at 10 days after the $1^{\text {st }}$ spray application. It is worth mentioning that these insecticides had maintained the larval CLM population at its lowest levels throughout the experimental period (Table $1 \& 2$ ).

\section{Discussion}

Generally, M. azedarach fruit extracts exert various effects on leafminer species. The population of the Agromyzid pea leafminer Liriomyza huidobrensis (Blanchard) was reduced after surface application of Melia fruit extract on swisschard and cucumber plants grown under field and greenhouse conditions, respectively (Abou-Fakhr Hammad et al. 2000a, 2000b). Similarly, M. azedarach immature fruit extracts resulted in fewer initiated larval mines of the vegetable leafminer Liriomyza sativae Blanchard on cowpea plants (Abou-Fakhr Hammad and McAuslane 2010). Furthermore, Melia extracts applied on various plants exerted toxic and repellent effect against the nymph and adult whiteflies of Bemisia species, respectively (Abou-Fakhr Hammad and McAuslane 2006; Abou-Fakhr Hammad et al. 2001; Abou-Fakhr Hammad et al. 2000a, 2000b).

In the current study, both Melia extracts behaved similarly as they yielded comparable results 10 days after the first spray application but unfortunately, the Melia mature fruit extract could not be tested thereafter. Extracts of different ages of Melia fruits were comparable in their effect against the CLM after the $1^{\text {st }}$ spray application similar to extracts of different ages of Melia fruits and leaves tested against B. tabaci (Abou-Fakhr Hammad et al. 2001). The significantly lower number of CLM live larvae in both fruit extract treatments in comparison with the control (Tables 1 \& 2), could have been caused by the ingestion of the Melia components present in the leaf mines. The fact that the number of larvae remained relatively constant after the second spraying with the green Melia extract may indicate a potential toxic or antifeedant effect of this extract. In comparison with the two biorational treatments, however, both Melia extracts were less efficient after 10 days of the first application and this efficiency did not improve even after the second spray of the Melia green fruit extract. This could be an indication of either low concentrations of the extracts reaching the mines or the result of a weakening effect of the active ingredients of the Melia extracts in the plant tissues with time. A weakening effect of azadirachtin (of initially $0.17 \mathrm{~g}$ active ingredient/cm trunk diameter) against the litchi stink bug pest, Tessaratoma papillosa Drury, in the plant tissues after about 2-3 weeks was reported by Schulte et al. (2006). Furthermore, the persistence of azadirachtin was prolonged in systemic applications compared with its residual life (4-8 days) when foliar sprayed (applied in the current study) under field conditions (Ascher 1993). In addition, the Melia extracts used in this study may have lacked the rapid penetration and translaminar action through the leaf tissues; characteristics of neem oil and abamectin, respectively (Mujica et al. 2000).

Different commercial insecticides including botanicals and other biorationals have been tested for the control of CLM. These products differ in their formulation and concentration of the active ingredients. Azadirachtin (as Margson- $\mathrm{O}^{\circledR} ; 25 \%$ a.i.), abamectin and mineral oil had a comparable effect against CLM (L1, L2 \& L3) on navel oranges under field conditions after $2^{\text {nd }}$ spray application (Abou-Fakhr Hammad and Antar 2003). Howard (1993) reported similar CLM results on Lime seedlings treated with the two biorationals, Avid ${ }^{\circledR}$ (abamectin 0.15 EC) and Azatin ${ }^{\circledR}$ (60 ppm azadirachtin) with Triton, at the flushing period. Plants sprayed with abamectin, however, remained completely free of leafminer damage, whereas 33 and $50 \%$ of azadirachtin treated leaves showed 
incipient mines 3 and 4 weeks post spraying, respectively; the incipient mines did not progress when observed 5 weeks post spraying with no leaf curling detected. These results indicate that botanical products including Melia plant extracts need more time to reveal their insecticidal effects compared with other biorational or synthetic pesticides that are known to have quick knock down action. Furthermore, the extract application efficiency could be enhanced by incorporating mineral oils that improve plant coverage and penetration of chemicals into leaf surfaces (Bográn et al. 2006); knowing that Melia extracts were found to be enhanced by Tween-20 when applied against B. tabaci on tomato plants (Jazzar and Abou-Fakhr Hammad 2003).

The compatibility of any potential and emerging pesticide with natural enemies is a basic component in building up Integrated Pest Management (IPM) programs. Villanueva-Jimenez and Hoy (1998) studied the relative toxicity of certain biorational pesticides to the CLM and its parasitoid Ageniaspis citricola using several bioassay methods. They classified azadirachtin $\left(\right.$ Neemix $\left.{ }^{\circledR}\right)+$ oil, and oil alone (FC 435-66) as IPMcompatible insecticides, while azadirachtin $\left(\operatorname{Align}^{\circledR}\right)+$ oil and neem oil $\left(\right.$ Neemgard $\left.^{\circledR}\right)$ were ranked as semicompatible insecticides, but avermectin (Agri-Mek $\left.{ }^{\circledR}\right)+$ oil and imidacloprid (Provado ${ }^{\circledR}$ ) when applied as sprays were considered as IPM-incompatible insecticides. The current results suggest that the Melia extract lends itself as a potential plant derived insecticide and merits further investigation in order to classify it as IPM compatible and use it with other control methods. The incorporation of these plant extracts might enhance the effect of natural enemies of the CLM under IPM programs; knowing that several predators and parasitoids (Karamaouna et al. 2010; Kalaitzaki et al. 2011; Tsagkarakis et al. 2013) were found to be important in reducing peak populations of the CLM, but apparently may need to be used in large numbers to provide good control of the pest (Knapp et al. 1995; Hoy and Nguyen 2006; Heppner and Fasulo 2010). Limited or no information is available not only on the natural enemies of the CLM in Lebanon, but also on the effect of botanical extracts on these beneficials. Melia extracts, however, have been tested against different natural enemy species. Percent parasitism of Diglyphus isaea (Walker) (Hymenoptera: Eulophidae), known to lower the populations of the leafminer L. sativae (Diptera: Agromyzidae) was not affected by Melia fruit extracts (Abou-Fakhr Hammad and McAuslane 2010). The same extracts were also found to have an additive effect on the whitefly Bemisia argentifolii Bellows \& Perring nymphal mortality when combined with the parasitoid Eretmocerus rui Zolnerowich and Rose (Abou-Fakhr Hammad and McAuslane 2006). Furthermore, Melia extracts were found to be harmless to the predator, Camptotylus reuteri (Jakovlev), of $B$. tabaci as these extracts did not cause any mortality of the predator (Jazzar and AbouFakhr Hammad 2003). Thus, Melia extracts deserve further investigations taking into consideration the locally available CLM natural enemies in order to be used in IPM programs.

In conclusion, this work showed that $M$. azedarach extracts clearly had adverse effects on the $P$. citrella by decreasing the number of live CLM larvae (L1 and L2 \& L3). These extracts are promising to be used in sustainable agriculture approaches against the CLM and deserve further investigations to fully elucidate their potential use alone and with biological control agents of this pest.

\section{Competing interests}

The authors declare that they have no competing interests.

\section{Authors' contributions}

EMA-FH proposed the topic, specified the required design and chronological steps of the experimental study, followed up on the execution of the experiment, participated in the statistical analysis of the data and adjusted the manuscript draft according to publication needed criteria. MMM carried out the experimental study (field and laboratory work), collected and tabulated the data for the statistical analysis and provided first draft of the manuscript. MTF analyzed the data and helped in their interpretation; also contributed with the corresponding author to the correction of the last revision of the manuscript. All authors read and approved the final manuscript.

\section{Acknowledgement}

The authors would like to express their gratitude to Mr. Abbas Mckenna, for his permission to proceed with this study on his citrus lemon orchard.

\section{Author details}

${ }^{1}$ Department of Plant Protection, Faculty of Agriculture and Veterinary Sciences, Lebanese University, Beirut, Lebanon. ${ }^{2}$ Department of Agricultural Sciences, Faculty of Agricultural and Food Sciences, American University of Beirut, Beirut, Lebanon. ${ }^{3}$ Department of Animal \& Veterinary Sciences, Faculty of Agricultural and Food Sciences, American University of Beirut, Beirut, Lebanon.

Received: 30 November 2012 Accepted: 25 March 2013 Published: 5 April 2013

\section{References}

Abou-Fakhr Hammad EM, Nemer NM, Hawi ZK, Hanna LT (2000a) Responses of the sweetpotato whitefly, Bemisia tabaci, to the Chinaberry tree (Melia azedarach L.) and its extracts. Ann Appl Biol 137:79-88 Abou-Fakhr Hammad EM, Nemer NM, Kawar NS (2000b) Efficacy of the Chinaberry tree (Meliaceae) aqueous extracts and certain insecticides against the pea leafminer (Diptera: Agromyzidae). J Agric Sci Vol 134:413-420

Abou-Fakhr Hammad EM, Zournajian H, Talhouk S (2001) Efficacy of extracts of Melia azedarach $\mathrm{L}$. callus, leaves and fruits against adults of the sweetpotato whitefly Bemisia tabaci (Hom. Aleyrodidae). J Appl Entomol 125:483-488 Abou-Fakhr Hammad EM, Antar NM (2003) Efficacy of selected insecticides on Phyllocnistis citrella Stainton (Lepidoptera: Gracillariidae) in navel orange (Citrus sinensis L.). Tests Agrochem Cultivars 24:6-7

Abou-Fakhr Hammad E, McAuslane H (2006) Effect of Melia azedarach L. Extract on Bemisia argentifolii (Homoptera: Aleyrodidae) and its Biocontrol Agent Eretmocerus rui (Hymenoptera: Aphelinidae). Environ Entomol 35(3):740-745

Abou-Fakhr Hammad E, McAuslane H (2010) Effect of Melia azedarach L. Extract on Liriomyza sativae (Diptera: Agromyzidae) and its Biocontrol Agent Diglyphus isaea (Hymenoptera: Eulophidae). J Food, Agric Environ 8(3\&4):1247-1252 Al-Akhras HA (2010) Effect of Melia azedarach on Black Aphid Aphis fabae, M.S. Thesis. Lebanese University, Beirut, Lebanon 
Amiri-Besheli B (2008) Efficacy of Bacillus thuringiensis, mineral oil, insecticidal emulsion and insecticidal gel against Phyllocnistis citrella Stainton (Lepidoptera: Gracillariidae). Plant Protec Sci 44(2):68-73

Amiri-Besheli B (2009) Toxicity evaluation of Tracer, Palizin, Sirinol, Runner and Tondexir with and without mineral oils on Phylocnistis citrella Stainton. Afr J Biotechnol 8(14):3382-3386, http://www.academicjournals.org/ajb/PDF/ pdf2009/20Jul/Amiri-Besheli.pdf

Amiri-Besheli B (2011) The Effect of Some Botanical Pesticides against Citrus Leafminer (CLM) and Two Spotted Mite (TSM). In: Stoytcheva M (ed) Pesticides in the Modern World - Pesticides Use and Management. InTech. doi:10.5772/950

Ascher KRS (1993) Nonconventional insecticidal effects of pesticides available from the neem tree, Azadirachta indica. Arch Insect Biochem Physiol 22:433-449

Ascher KRS, Schmutterer H, Zebitz CPW, Naqvi SNH (1995) The Persian lilac or Chinaberry tree: Melia azedarach L. In: Schmutterer H (ed) The Neem Tree: Source of Unique Natural Products for Integrated Pest Management, Medicine. Industry and Other Purposes. VCH, Weinheim, Germany, pp 605-642

Beattie A (2004) Citrus leafminer, 4th edn. NSW Department of Primary Industries, University of Western Sydney, Australia, http://www.dpi.nsw.gov.au/_data/ assets/pdf_file/0006/137634/citrus-leafminer.pdf

Bográn CE, Ludwig S, Metz B (2006) Using Oils as Pesticides. Texas A\&M University System; AgriLife Extension. E-419 11/06. http://insects.tamu.edu/ extension/publications/epubs/e-419.cfm

Dias C, Carsia P, Simoes N, Oliveira L (2005) Efficacy of Bacillus thuringiensis against Phyllocnistis citrella (Lepidoptera: Phyllocnitidae). J Econ Entomol 98(6):1880-1883

FAO - Food \& Agriculture Organization of the United Nations (1992) Pesticide residues in food. Report No. 116., p 146

Franzen H (1993) Need for development of new strategies for locust control. In: Rembold H (ed) New strategies for locust control 89:9-13. ATSAF, Bonn, Germany

Gomez KA, Gomez AA (1984) Statistical procedure for agricultural research. John Wiley and Sons, USA

Grainage M, Ahmed S (1988) Handbook of plants with pest-control properties. Wiley-Interscience, New York

Heppner JB, Fasulo TR (2010) Citrus Leafminer, Phyllocnistis citrella Stainton (Insecta: Lepidoptera: Phyllocnistinae). In: EENY-038 (IN165), one of a series of Featured Creatures from the Entomology and Nematology Department. Florida Cooperative Extension Service, Institute of Food and Agricultural Sciences, University of Florida, Florida, USA, http://edis.ffas.ufl.edu/pdffiles/IN/IN16500.pdf

Howard FW (1993) Effectiveness of Two Biorational Substances (Neem and abamectin) against Citrus Leaf Miner. TropicLine 6, No. 6. http://flrec.ifas.ufl. edu/pdfs/TropicLine/TropicLine_06-6a.pdf

Hoy MA, Nguyen R (2006) Classical Biological Control of the Citrus Leafminer in Florida. Gainesville. In: Florida: IPM Florida, Institute of Food and Agricultural Sciences (IFAS) Extension. University of Florida, IFAS, Entomology and Nematology Department, Gainesville, and Division of Plant Industry. http:// ipm.ifas.ufl.edu/agriculture/citrus/dmhoy7.shtml

Isman MB (2006) Botanical insecticides, deterrents, and repellents in modern agriculture and an increasingly regulated world. Annu Rev Entomol 51:45-66 Jacobson M (1975) Insecticides from plants: A review of the literature, 1954-1971. Agricultural Handbook 461. U.S Department of Agriculture, Washington, D.C, p 138

Jacobson M (1989) Botanical pesticides, past present and future. In: Arnason JJ, Philogen BR, Morand P (eds) Insecticides of Plant Origin. Proceedings of the American Chemical Society (ACS) Symposium Series, 387th edn. ACS, Washington, DC, USA, pp 1-10

Jazzar C, Abou-Fakhr Hammad E (2003) The efficacy of enhanced aqueous extracts of Melia azedarach leaves and fruits integrated with the Camptotylus reuteri releases against the sweetpotato whitefly nymphs. B Insectol 56(2):269-275

Jones J (2001) Citrus leafminer. University of Arizona, College of Agriculture \& Life Sciences, Arizona: USA, http://cals.arizona.edu/crops/citrus/insects/leafminer. html

Kalaitzaki AP, Tsagkarakis AE, Lykouressis DP (2011) Population fluctuation of Phyllocnistis citrella and its parasitoids in two citrus species in Western Crete (Greece). Entomologia Hellenica 20:31-44

Karamaouna F, Pascual-Ruiz S, Aguilar-Fenollosa E, Verdú MJ, Urbaneja A, Jacas JA (2010) Changes in predation and parasitism of the citrus leafminer Phyllocnistis citrella Stainton (Lepidoptera: Gracillariidae) populations in Spain following establishment of Citrostichus phyllocnistoides (Hymenoptera: Eulophidae). Biol Control 52:37-45
Kerns D, Wright G, Loghry J (2001) Citrus Leafminer (Phyllocnistis citrella). Cooperative Extension. The University of Arizona. http://ag.arizona.edu/crop/ citrus/insects/leafminer.pdf

Knapp JL, Albrigo LG, Browning HW, Bullock RC, Heppner JB, Hall DG, Hoy MA, Nyugen R, Peña JE, Stansly PA (1995) Citrus Leafminer, Phyllocnistis citrella Stainton: Current status in Florida-1994. Institute of Food and Agricultural Sciences, Florida Cooperative Extension Service, University of Florida, Gainesville, Florida, USA, p 26

Lowery DT, Isman MB (1994) Effects of Neem and azadirachtin on Aphids and their Natural Enemies. In: Hedin PA (ed) Bioregulators for Crop Protection and Pest Control, ACS Symposium Series 557. American Chemical Society, Washington, D.C. USA, Chapter 7:78-91

Lydon J, Duke SO (1989) Potential of plants for pesticide use. Herbs, spices and medicinal plants: Recent advances in botany, horticulture, and pharmacology, vol. 4. Oryx Press, Phoenix, Arizona, USA

Miller F, Uetz S (1998) Evaluating Biorational Pesticides for Controlling Arthropod Pests and their Phytotoxic Effects on Greenhouse Crops. HortTechnology 8 (2):185-192

Moussa Z, El Hajj AK (2010) Citrus production in Lebanon Pre- feasibility Study of IPM of Citrus, Lebanese Agriculture Research Institute (LARI) - Lebanon. IAM - Bari, Italy, pp 1-10

Mujica N, Pravatiner M, Cisneros F (2000) Effectiveness of abamectin and plant-oil mixtures on eggs and larvae of the leafminer fly, Liriomyza huidobrensis Blanchard. CIP Program Report 1999-2000:161-166

Nardo EAB, Costa AS, Lourencao LA (1997) Melia azedarach extract as an antifeedant to Bemisia tabaci (Homoptera: Aleyrodidae). Fla Entomol 80:92-94

SAS Institute (1992) SAS User's Guide: Statistics, 5th edn. SAS Institute Inc., Cary, NC, USA

Schulte MJ, Martin K, Sauerborn J (2006) Effects of azadirachtin injection in litchi trees (Litchi chinensis Sonn.) on the litchi stink bug (Tessaratoma papillosa Drury) in northern Thailand. J Pest Sci 79:241-250

Schmutterer H (1990) Properties and potential of natural pesticides from the neem tree, Azadirachta indica. Annu Rev Entomol 35:271-297

Shin-Foon C (1987) Experiments on the practical application of chinaberry, Melia azedarach, and other naturally occurring insecticides in China. In: Schumutterer $H$, Ascher KRS (eds) Natural Pesticides from the Neem Tree and Other Tropical Plants. GTZ Press, Eschborn, Federal Republic of Germany, pp 661-668

Tsagkarakis AE, Kalaitzaki AP, Lykouressis DP (2013) Phyllocnistis citrella Stainton (Lepidoptera: Gracillariidae) and its parasitoids on three citrus varieties in Greece. Phytoparasitica 41(1):23-29. doi:10.1007/s12600-012-0258-9

Valladares G, Defagó MT, Palacios SM, Carpinella MC (1997) Laboratory evaluation of Melia azedarach (Meliaceae) extracts against the elm leaf beetle (Coleoptera: Chrysomelidae). J Econ Entomol 90:747-750

Villanueva-Jimenez JA, Hoy MA (1998) Toxicity of pesticides to the citrus leafminer and its parasitoid Ageniaspis citricola evaluated to assess their suitability for an IPM program in citrus nurseries. BioControl 43:357-388

Weinzierl R, Henn T (1991) Alternatives in Insect management: Biological and Biorational Approaches. North Central Regional Extension, Publication 401. Cooperative Extension Service. University of Illinois at Urbana-Champaign, Illinois, USA, http://web.aces.uiuc.edu/vista/pdf_pubs/altinsec.pdf

Yang RZ, Tang CS (1988) Plants used for pest control in China: A literature review. Econ Bot 42:376-406

\section{doi:10.1186/2193-1801-2-144}

Cite this article as: Mckenna et al:: Effect of Melia azedarach (Sapindales:

Meliaceae) fruit extracts on Citrus Leafminer Phyllocnistis citrella

(Lepidoptera: Gracillariidae). SpringerPlus 2013 2:144. 